\title{
Investigation of Defect Properties in Titanium Nitride Obtained by Laser Sintering
}

\author{
${ }^{1-4}$ E.A. Konstantinova, ${ }^{1}$ A.S. Ilin, ${ }^{1-3}$ A.V. Pavlikov and ${ }^{1,3,5}$ P.A. Forsh \\ ${ }^{1}$ Faculty of Physics, M.V. Lomonosov Moscow State University, Leninskie Gory 1-2, \\ 119991 Moscow, Russia \\ ${ }^{2}$ Federal State-Owned Unitary Enterprise, \\ "All-Russian Research Institute for Optical and Physical Measurements", \\ Ozernaya St 46, 119361 Moscow, Russia \\ ${ }^{3}$ National Research Center "Kurchatov Institute", Akademika Kurchatova 1, 123182 Moscow, Russia \\ ${ }^{4}$ Department of Nano, Bio, Information Technology and Cognitive Science, \\ Moscow Institute of Physics and Technology, Institutskij 9, Dolgoprudny, \\ 141701 Moscow Region, Russia \\ ${ }^{5}$ P.N. Lebedev Physical Institute of the Russian Academy of Sciences, Leninsky Prospekt 53, \\ 119991 Moscow, Russian Federation, \\ as.ilin@physics.msu.ru
}

\begin{abstract}
We present an investigation of titanium nitride samples obtained by additive technologies using EPR-spectroscopy. It was established that the main type of defects in this material is nitrogen atoms with unpaired electrons ( $\mathrm{N}$-radicals). It was found that the defect concentration increases by two times under illumination. This effect is reversible which is evidenced by the charge exchange of the N-centers. The data obtained may be useful for producing coatings based on the investigated samples.
\end{abstract}

Key words: Titanium nitride, laser sintering, N-centers, N-radicals, concentration, nitrogen

\section{INTRODUCTION}

In recent years, there has been an increase in the publications devoted to the description of the basic principles of additive technologies and to the investigation of materials produced by these technologies (Groza et al., 2000; Pierson, 1996; Babakova et al., 2015; Penilla and Wang, 2008; Jeyachandran et al., 2007; Chung et al., 2004). The influence of parameters of manufacturing on structural, electrophysical properties and phase composition is widely studied (Pierson, 1996; Jeyachandran et al., 2007; Chung et al., 2004; Shishkovsky, 2001; Saprykina et al., 2011; Gureev et al., 2000). The prospects of their application in the field of consumer goods, electronics, automotive industry, medicine, aerospace industry, industrial engineering and other related fields are discussed (Pierson, 1996; Panchenko et al., 2009). One of the effective methods of obtaining additive products is laser sintering (Babakova et al., 2015; Jeyachandran et al., 2007; Chung et al., 2004; Shishkovsky, 2001; Saprykina et al., 2011; Gureev et al., 2000). The samples formed in this process have a number of unique properties and low cost in comparison with their analogues, obtained with the help of conventional methods of synthesis (Jeyachandran et al.,
2007; Chung et al., 2004; Shishkovsky, 2001). Their phase composition and structural properties are studied in detail. Data on the nature and properties of defects in such objects are practically absent. In this study, titanium nitride obtained by laser sintering was chosen as the object of investigation. It is known that titanium-containing materials are characterized by the presence of a large number of point defects that are paramagnetic which makes it possible to study their properties by the method of Electron Paramagnetic Resonance (EPR) spectroscopy (Le et al., 2015; Tarasov et al., 2015; Kokorin and Bahnemann, 2003). Therefore, in order to identify these defects and to determine their main parameters, we used this method and the technique developed on the basis of this method to diagnose the radicals formed in the process of various physicochemical effects in the "in situ" mode in the samples obtained with the help of additive technologies.

\section{MATERIALS AND METHODS}

As an additive technology for the formation of investigated samples of titanium nitride, laser sintering using an Nd: YAG laser was used. The titanium powder of

Corresponding Author: A.S. Ilin, Faculty of Physics, M. V. Lomonosov Moscow State University, Leninskie Gory 1-2, 119991 Moscow, Russia, as.ilin@physics.msu.ru, +7(495)939-16-66 
the PTS grade with a fraction not higher than $100 \mu \mathrm{m}$ was pre-dried and sieved. Further, it was subjected to mechanical grinding in a FRITSCH Planetary Mill "Pulverisette 7" for $20 \mathrm{~min}$. Sintering of the ground titanium powder was carried out in a special chamber at nitrogen pressure $15 \mathrm{~atm}$ using a $17 \mathrm{~W}$ laser radiation power. The scanning speed of the laser beam was $2 \mathrm{~cm} / \mathrm{sec}$.

The EPR spectra were measured with an EPR spectrometer Bruker ELEXSYS-500 (operating frequency $9.5 \mathrm{GHz}$, sensitivity $5-10^{10} \mathrm{spin} / \mathrm{Gs}$ ). Illumination of the samples was carried out directly in the cavity of the spectrometer using a Bruker ER 202 UV mercury lamp (power of $50 \mathrm{~W}$ ) in a wide spectral range $(270-900 \mathrm{~nm}$ ). The measurements were carried out over a wide temperature range from $30-300 \mathrm{~K}$ using the Bruker ER $4112 \mathrm{HV}$ temperature control system. To calculate the concentration of paramagnetic centers, we used a standard $\mathrm{CuCl}_{2}\left(2 \mathrm{H}_{2} \mathrm{O}\right)$ with a known number of spins. Computer simulation of the EPR signal was carried out in the EasySpin Software module of the MATLAB package (Stoll and Schweiger, 2006).

The morphology of the samples was examined by scanning electron microscopy using the LEO 1420 microscope. Additional information was extracted from XPS measurements using Axis Ultra DLD (Kratos, UK) spectrometer equipped with a monochromatic $\mathrm{Al} \mathrm{K} \alpha$ $\mathrm{X}$-ray source.

\section{RESULTS AND DISCUSSION}

According to the electron microscopy investigation TiN samples have porous structure and consist of agglomerates, the size of which does not exceed $1 \mu \mathrm{m}$ (Fig. 1). XPS measurements showed that the samples contain $77.8 \mathrm{wt} \%$ of Ti and $22.2 \mathrm{wt} . \%$ of N.

Figure 2 shows the EPR spectra of titanium nitride samples measured under dark conditions and in the presence of illumination. As follows from the presented data, the EPR spectrum has a complex structure, therefore, to properly interpret the results, computer simulation is required. Figure 3 shows the results of computer simulations of the experimental EPR spectrum. The following values of the g-tensor and the Hyper Fine interaction Tensor (HFT) were obtained: $\mathrm{g}_{1}=2.0041$, $\Delta \mathrm{H}_{1}=3.3 \mathrm{G} ; \mathrm{g}_{2}=2.0028, \Delta \mathrm{H}_{2}=1.9 \mathrm{G} ; \mathrm{g}_{3}=2.0021$, $\Delta \mathrm{H}_{3}=2.7 \mathrm{G}$ and accordingly, $\mathrm{A}_{1}=1.9 \mathrm{G}, \mathrm{A}_{2}=3.1 \mathrm{G}$, $\mathrm{A}_{3}=32.1$ Gs. The EPR signal with these anisotropic parameters can be attributed to the paramagnetic centers which are nitrogen atoms (the spin of the nucleus $\mathrm{I}=1$ ) with an unpaired electron ( $\mathrm{N}$-radicals), according to the literature by Le et al. (2015), Tarasov et al. (2015), Kokorin and Bahnemann (2003).

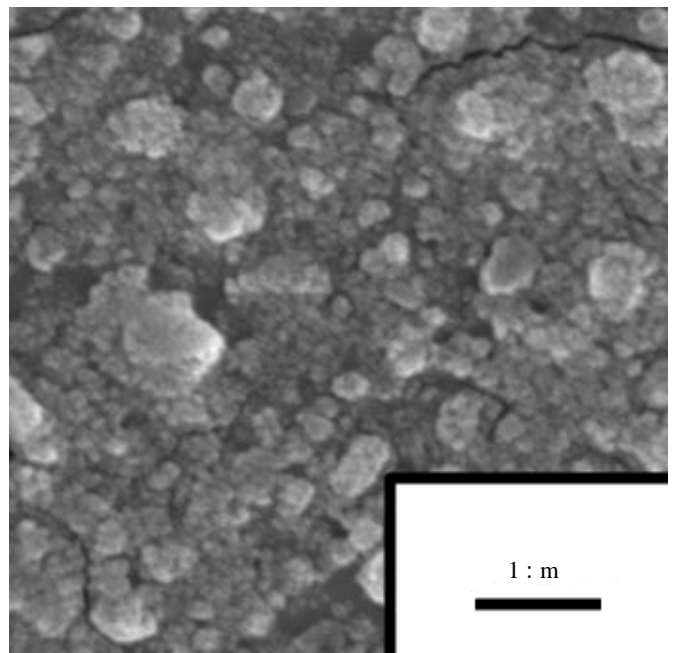

Fig. 1: SEM image of TiN samples

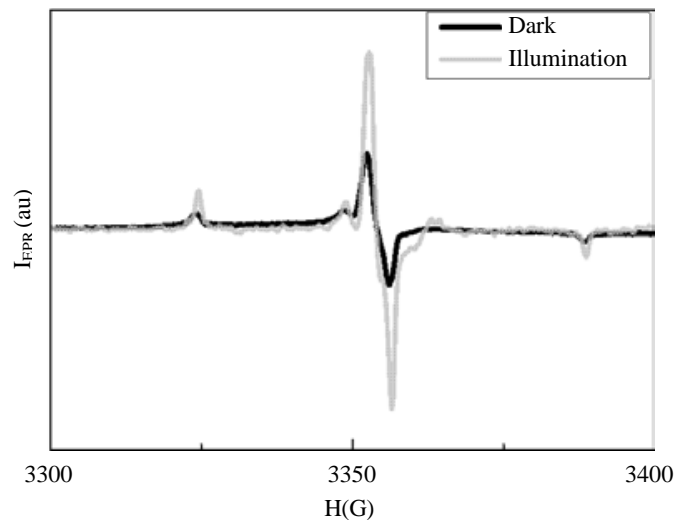

Fig. 2: EPR spectrum of titanium nitride obtained by laser sintering in the dark and under illumination $(\mathrm{T}=300 \mathrm{~K})$

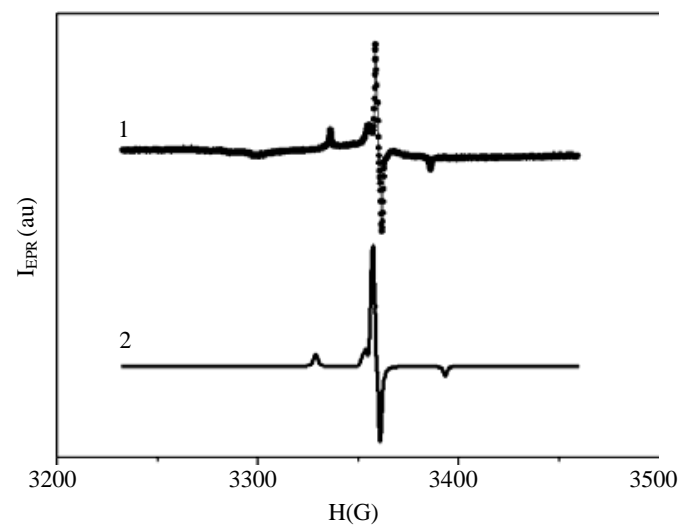

Fig. 3: Experimental and theoretical EPR spectra of titanium nitride obtained by laser sintering $(\mathrm{T}=300 \mathrm{~K})$ 


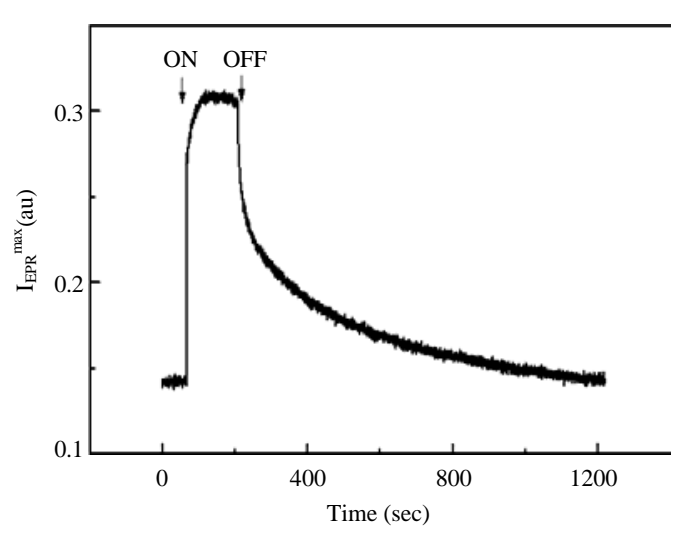

Fig. 4: Variations in the amplitude of the EPR signal in the dark-illumination-dark cycle. The arrows indicate the moments of switching on and off the illumination $(\mathrm{T}=300 \mathrm{~K})$

In addition, the analysis in Fig. 2 shows that when illuminated, the amplitude of the EPR signal increases (Fig. 2) which indicates an increase in the concentration of the paramagnetic centers. The calculated N-center concentration in the samples studied was $\mathrm{Ns}=8 \times 10^{16} \mathrm{~cm}^{-3}$ in the dark and $\mathrm{Ns}=1.8 \times 10^{17} \mathrm{~cm}^{-3}$ under illumination for $5 \mathrm{~min}$. The illumination effect was reversible. This is evidenced by the measured kinetics of the relaxation of the amplitude of the EPR signal (Fig. 4). The reversibility of the illumination effect can be explained by charge exchange of N-radicals. Indeed, prior to illumination in the sample, there are probably both paramagnetic centers (containing an unpaired electron) and non-paramagnetic centers (containing two coupled electrons). During illumination, photoexcitation of electrons from non-paramagnetic centers propably occurs, the remaining defects become centers with one electron, i.e., become paramagnetic which leads to an increase in the amplitude of the EPR signal and the concentration of N-centers, respectively (Fig. 4). After the illumination ceases, the reverse process takes place-the capture of photoexcited electrons by paramagnetic defects and their transition to a nonparamagnetic state.

It should be noted that a decrease in the temperature down to $30 \mathrm{~K}$ did not reveal any new signals in the EPR spectrum. EPR measurements at low temperatures were performed in order to find other paramagnetic centers in the structure of our samples, characterized by short spin-lattice relaxation times and accordingly, wide EPR lines (according to the Heisenberg uncertainty principle) that are not detected at room temperatures (Weil and Bolton, 2007).
Thus, in this research, the samples of titanium nitride obtained by laser sintering were studied using the EPR spectroscopy method of in-situ diagnostics of radicals. These radicals were formed in the course of various physicochemical effects in samples fabricated by additive technologies. It was shown that this material contains paramagnetic centers which are nitrogen atoms with an unpaired electron (N-radicals).

\section{CONCLUSION}

The concentration of these defects was determined, the value of which reversibly changed in the cycle: dark-illumination-dark which indicates the processes of charge exchange of defects in the samples under study. The information obtained is important both from the fundamental point of view of studying the electronic properties of new materials and also from practical one, since, point defects substantially influence the electrophysical properties of the samples.

\section{ACKNOWLEDGEMENTS}

This research was performed with the financial support of the Ministry of Education and Science of Russia under Agreement No. 14.625.21.0041 of September 26, 2017 (unique identifier RFMEFI62517X0041). The experiments were performed using the facilities of the Collective Use Center at the Faculty of Physics of Moscow State University.

\section{REFERENCES}

Babakova, E.V., A.V. Gradoboev, A.A. Saprykin, E.A. Ibragimov and V.I. Yakovlev et al., 2015. Comparison of activation technologies powder ECP-1 for the synthesis of products using SLS. Appl. Mech. Mater., 756: 220-224.

Chung, K.H., G.T. Liu, J.G. Duh and J.H. Wang, 2004. Biocompatibility of a Titanium-Aluminum nitride film coating on a dental alloy. Surf. Coat. Technol., 188: 745-749.

Groza, J.R., J.D. Curtis and M. Kramer, 2000. Field-Assisted sintering of nanocrystalline titanium nitride. J. Am. Ceram. Soc., 83: 1281-1283.

Gureev, D.M., R.V. Ruzhechko andI.V. Shishkovskii, 2000. Selective laser sintering of PZT ceramic powders. Tech. Phys. Lett., 26: 262-264.

Jeyachandran, Y.L., S. Venkatachalam, B. Karunagaran, S.K. Narayandass and D. Mangalaraj et al., 2007. Bacterial adhesion studies on titanium, titanium nitride and modified hydroxyapatite thin films. Mater. Sci. Eng. C., 27: 35-41. 
Kokorin, A.I. and D. Bahnemann, 2003. Chemical Physics of Nanostructured Semiconductors. 1st Edn., CRC Press, Boca Raton, Florida, USA., ISBN: 9781498708630, Pages: 264.

Le, N.T., E.A. Konstantinova, A.I. Kokorin, T. Kodom and N. Alonso-Vante, 2015. Recharge processes of paramagnetic centers during illumination in nitrogen-doped nanocrystalline titanium dioxide. Chem. Phys. Lett., 635: 241-244.

Panchenko, V.Y., V.S. Golubev, V.V. Vasiltsov, M.G. Galushkin and V.D. Dubrov et al., 2009. [Laser Technologies for Processing Materials: Modern Problems of Fundamental Research and Applied Development]. Fizmatlit Publisher, Moscow, Russia, ISBN:978-5-9221-1023-5, Pages: 704 (In Russian).

Penilla, E. and J. Wang, 2008. Pressure and temperature effects on stoichiometry and microstructure of nitrogen-rich $\mathrm{TiN}$ thin films synthesized via. reactive magnetron DC-Sputtering. J. Nanomater., 2008: 1-9.
Pierson, H.O., 1996. Handbook of Refractory Carbides and Nitrides: Properties, Characteristics, Processing and Applications. Noyes Publications, New Jersey, ISBN: 13- 9780815513926 , Pages: 340.

Saprykina, N.A., A.A. Saprykin and D.A. Shigaev, 2011. Investigating factors influencing the quality of a surface obtained by laser sintering. Met. Process., 4 : 78-82.

Shishkovsky, I.V., 2001. Synthesis of functional products from gradient materials by the method of selective laser sintering. J. Perspektivnye Mater., 5: 60-64.

Stoll, S. and A. Schweiger, 2006. EasySpin, a comprehensive software package for spectral simulation and analysis in EPR. J. Magn. Reson., 178: $42-55$

Tarasov, A., A. Minnekhanov, G. Trusov, E. Konstantinova and A. Zyubin et al., 2015. Shedding light on aging of N-Doped titania photocatalysts. J. Phys. Chem. C., 119: 18663-18670.

Weil, J.A. and J.R. Bolton, 2007. Electron Paramagnetic Resonance: Elementary Theory and Practical Applications. 2nd Edn., John Wiley \& Sons, Hoboken,New Jersey, USA., ISBN: 978-0471-75496-1, Pages: 668 . 